\title{
Advertising Liking Recognition Technique Applied to Neuromarketing by Using Low-Cost EEG Headset
}

\author{
Luis Miguel Soria Morillo ${ }^{1}$, Juan Antonio Alvarez García ${ }^{1}$, \\ Luis Gonzalez-Abril ${ }^{2}$, and J.A. Ortega Ramirez ${ }^{1}$ \\ ${ }^{1}$ Computer Languages and Systems Dept., \\ University of Seville, 41012 Seville, Spain \\ 2 Applied Economics I Dept., \\ University of Seville, 41018, Seville, Spain \\ \{lsoria, jaalvarez, luisgon, jortega\}@us.es \\ http://www.us.es
}

\begin{abstract}
In this paper a new neuroscience technique is applied into Marketing, which is becoming commonly known as the field of Neuromarketing. The aim of this paper is to recognize how brain responds during the visualization of short advertising movies. Using low cost electroencephalography (EEG), brain regions used during the presentation have been studied. We may wonder about how useful it is to use neuroscience knowledge in marketing, what can neuroscience add to marketing, or why use this specific technique. By using discrete techniques over EEG frequency bands of a generated labeled dataset, C4.5 and ANN learning methods have been applied to obtain the score assigned to each ads by the user. This techniques allows to reach more than $82 \%$ of accuracy, which is an excellent result taking into account the kind of low-cost EEG sensors used.
\end{abstract}

Keywords: neuromarketing, electroencephalography, advertising, brain, eeg, brain-computer interaction.

\section{Introduction}

The use of electroencephalography has been used over the last decade for the study of brain activity. In most of the studies is to determine the effects of certain thoughts on the cerebral cortex. Thus a cause-effect relationship that explores the different cerebral cortex is established. The combination of medicine and learning systems have been able to achieve milestones that were unthinkable years. Since the isolation of brain activity based on the thoughts and your use of electronic devices through a brain interface. This has led to a new era in the field of interaction human-computer, characterized by the absence of direct orders or actions by the user, to make way for management through direct thoughts. In an effort to isolate the actions, researchers have found associations for determining whether the music is heard or not the user's taste, or if a TV ad 
captures or not the individual's attention. This application is being exploited by the advertising industry. These actions, in this special paper attention will be paid to the relationship between the display of advertisements short video and the effects they cause on the mental state of the user. Specifically, this study aims to provide a solution to a recurring problem in the advertising field, as is determining cortical level marketing preferences without user interaction How does the cerebral cortex a given ad is user friendly? Are there effects electric level when the user is viewing advertising content relevant to your tastes? Determining these partnerships will allow high-level go a step further in neuromarketing [Lee et al.(2007)Lee, Broderick, and Chamberlain, Ariely and Berns(2010), Morin(2011)]. So far, most studies in the field of neuromarketing have been based on the use of electroencephalography sensors or sensors installed galvanic skin resistance of the user to determine the impact of ads brainwave level isolation [Senior and Lee(2008), Murphy et al.(2008)Murphy, Illes, and Reiner]. However, there have been few studies that have used this technology to classify information binomial how classes [I like] or [I hate]. This first step described in this article allow the development of a learning system based on neural networks [Hagan et al.(1996)Hagan, Demuth, Beale, et al., Dreiseitl and Ohno-Machado(2002)] and decision trees (C4.5) [Quinlan(1993)] to determine patterns of classification of content. These patterns, in a second level, given the possibility to determine independently whether an ad has not liked or user. The study of cortical signals at a high level can be transcendent to establish direct and automatic relationship between tastes and mental reflexes, which will make the user environment to suit user preferences [Morin(2011)].

\section{Related Works}

The first psychological studies done using EEG date as far as 1979. His studies and others later validated that electrical patterns were lateralized in the frontal region of the brain. Generally, the measure of alpha-band waves $(813 \mathrm{~Hz})$ in the left frontal lobe indicates positive emotions. A strong involvement of parietal areas during the observation of the TV commercials with an affective and cognitive content was also noted in a previous study, performed by using sophisticated MEG recordings. The magneto field tomography (MFT) results showed an increasing activity during the observation of cognitive stimuli rather than affective commercials in parietal and superior prefrontal areas. These regions are known to be associated with executive control of working memory and maintenance of highly processed representation of complex stimuli. Although the affect-related activations are more variable across subjects, these findings are consistent with previous PET and fMRI studies showing that stimuli with affective content modulates activity in the orbitofrontal and retrosplenial cortex, amygdale, and brainstem. However, in this study is to use a low-resolution sensor and an absequible price for this task [Vecchiato et al.(2011) Vecchiato, Astolfi, De Vico Fallani, Toppi, Aloise, Bez, Wei, Kong, Dai, Cincotti, et al.]. There are several startups that have products under development similar to Neurosky Mindwave (1) features, but with a much smaller 
size. Many of these products under development have a similar appearance to a wireless headphones, which makes the user can wear them continuously. This step in electroencephalograms wearable will require the use of simple devices and efficient algorithms to determine various aspects and relationships such as neuronal including a recognition of advertising tastes. There are also various applications of brain computer interfaces oriented to domestic use. Home automation and applications may be fostered by the massive use of this technology. Today, thanks to systems such as OSGi [Alliance(2003)] providers can connect context of this typology [Martín et al.(2009)Martín, Seepold, Madrid, Álvarez, Fernández-Montes, and Ortega, Martínez Fernández et al.(2010)Martínez Fernández, Seepold, Augusto, and Madrid, Soria-Morillo et al.(2011)Soria-Morillo, Ortega-Ramírez, GonzálezAbril, and Álvarez-García].

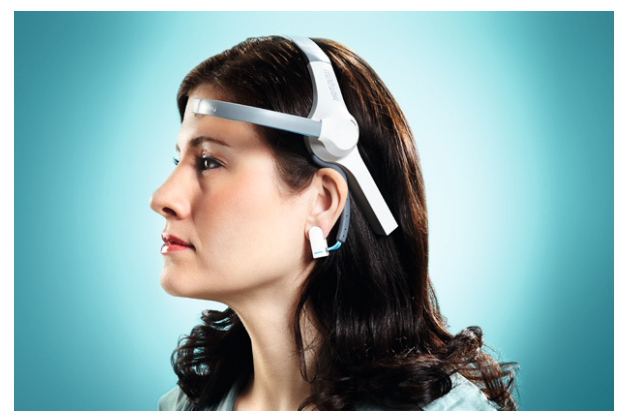

Fig. 1. Neurosky mindwave device

\section{Dataset}

In order to carry out the taste ads recognition system, a dataset was produced. This dataset has been carried out by displaying a set of 14 ads for a total of 10 people of different age and sex. The distribution of subjects in the experiment is shown in Table 1 .

The concentration level refers to the attitude presented by the person before conducting the test. This attribute can have, average high values (high interest in performing the test and little impact from external distractions) (high interest in performing the test and noticeable impact from external distractions) and low (average interest in conducting proof and high impact from external distractions). Users with different values in this attribute have been selected to test the robustness of face recognition patterns of middle- and high distraction, which are very common when users view the ads. The dataset was generated automatically by an Android app developed for this purpose. This application stores the videos which will be played. Once the videos have been played, the application prompts the user to issue a rating on them and, previously, indicating which video reminds (the latter can be understood as an indicator of the impact of the announcement on the user). The result is an XML file that contains the user's profile, 
Table 1. Subject profile on the data recovery process

\begin{tabular}{|l|l|l|l|}
\hline Subject & Age & Gender & $\begin{array}{l}\text { Concentration } \\
\text { level }\end{array}$ \\
\hline Subject 1 & 28 years & man & high \\
Subject 2 & 31 years & woman & medium \\
Subject 3 & 27 years & woman & medium \\
Subject 4 & 26 years & man & high \\
Subject 5 & 54 years & man & low \\
Subject 6 & 59 years & woman & medium \\
Subject 7 & 60 years & man & high \\
Subject 8 & 63 years & woman & low \\
Subject 9 & 42 years & man & high \\
Subject 10 & 46 years & woman & medium \\
\hline
\end{tabular}

Listing 1.1. content of dataset description XML

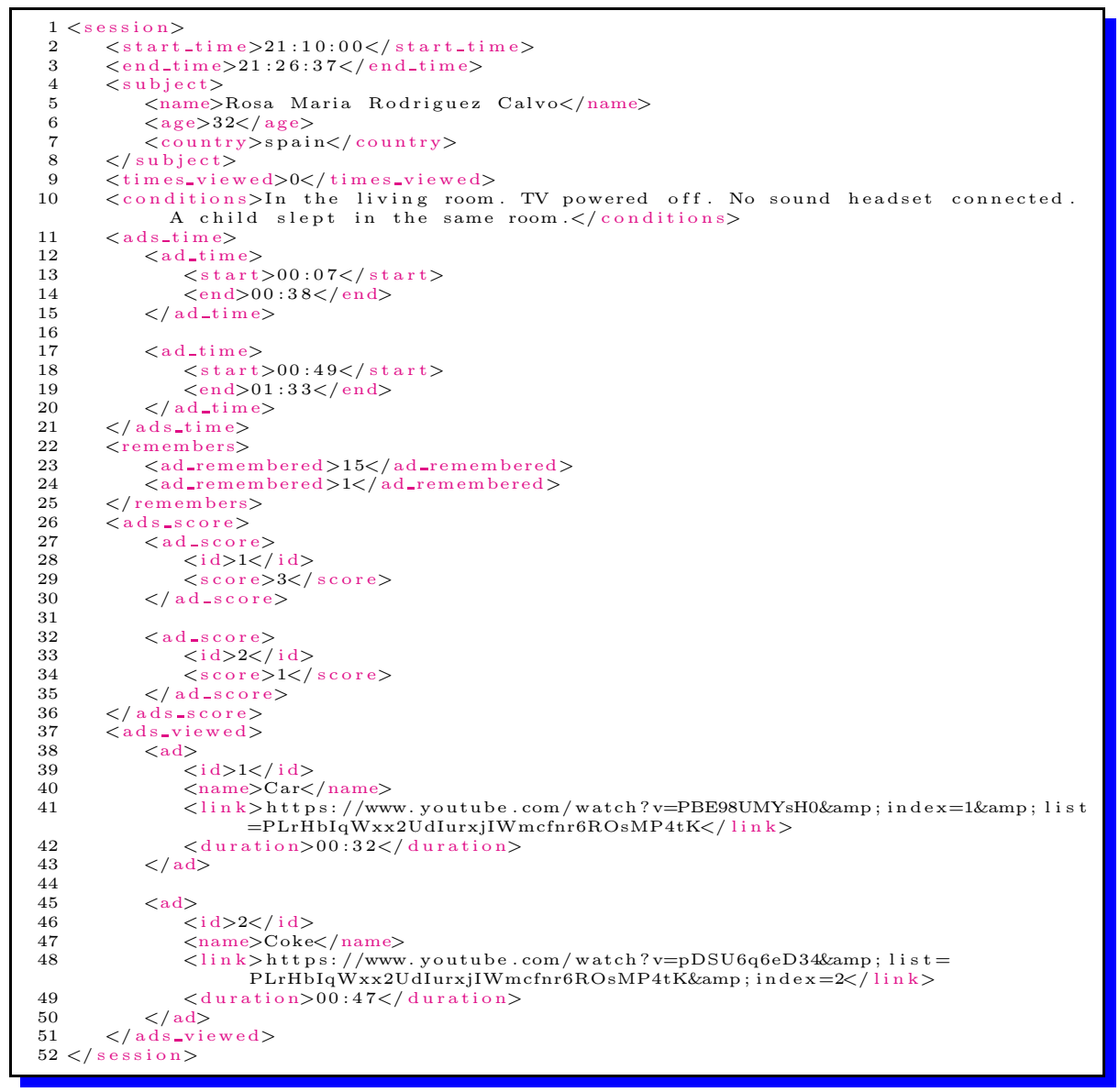


temporal information on the beginning and end of display ads, ads remembered by the user after completion of the test, score given for each ad and, finally, information for each ad. The score for each ad can be in the range $[0,5]$, with 0 being the minimum score (has not liked) and 5 high (liked it a lot). With this XML file, the information visualization session is stored. This information is important when learning process is conducting. On the other hand, while the user proceeds to displaying content, by Neurosky MindWave device information about brain activity associated is collected. All values delivered by the device are stored in order to obtain new statistics from them. The base signals are meditation, attention, delta amplitude, theta amplitude, low alpha amplitude, high alpha amplitude, low beta amplitude, high beta amplitude, low gamma amplitude and high gamma amplitude. This information can be seen in Figure 2 .

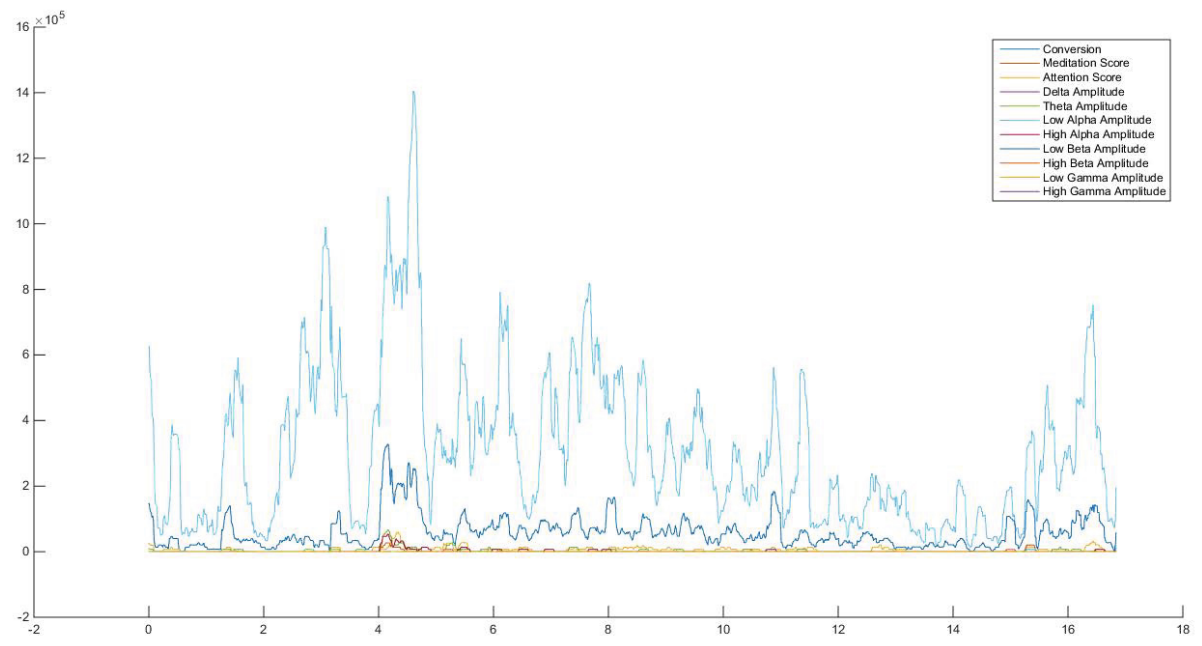

Fig. 2. EEG data from Neurosky Mindwave

From this database is possible to obtain a total of 480 statistical sampling parameters. These parameters will be considered hereafter as input variables for the classification system. Thanks to statistical signal derivatives from base values, discrete relationships can be established between instances with no temporary data processing needed. By one side, this allows to reduce the complexity of the learning and classification process and by other side, results will be more readable.

\section{Advertising Recognition Method}

In order to implement the recognition system a learning process on the data described above has been previously applied. With the purpose of allow comparison between different classification techniques, two methods have been studied: 

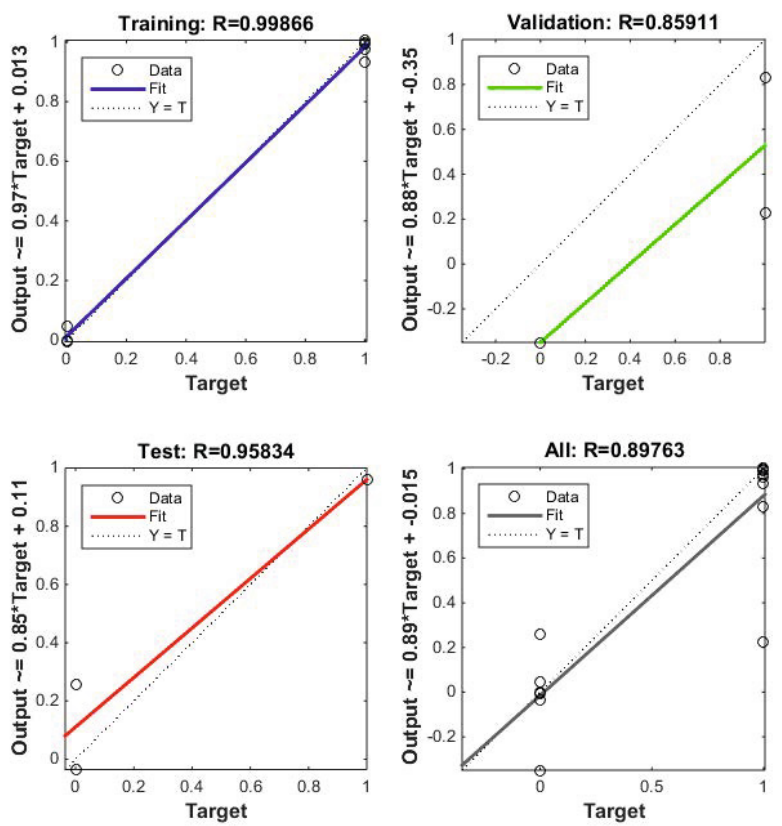

Fig. 3. Linear regression of targets relative to outputs

neural networks and decision tree. In the first case it has been decided to use a neural network where different statistical inputs are obtained from biometric parameters of the EEG, and targets are the score associated with each instance (advertising). For validation has established a set of 10 -folds cross validation with $70 \%$ of training data, $15 \%$ of validation and $15 \%$ of testing. The network configuration, as shown in Figure 4, is composed of two layers (one hidden and one output). The hidden layer consistes on five neurons, while the output layer has only one neuron. This configuration has been obtained from experiments and their results are shown in Figure 5. Sigmoid type of neurons have been employed in both layers, the hidden and the output layers. The algorithm used for training process was the Scaled Conjugate Gradient and performance is optimized by the function Cross-Entropy. In Figure 3 regression line during the learning process can be seen.

On the other hand, a decision tree for classifying instances has been used. In this case, the classification algorithm is C4.5 [Quinlan(1993)] was applied from the dataset of statistical inputs. The structure of the resulting tree is shown in Figure 6. Used this method, the accuracy is around $71 \%$. The testing and validation process employed in this case has been the same to the previous setting using ANN (Artificial Neural Networks) [Hagan et al.(1996)Hagan, Demuth, Beale, et al.], so the results can be compared.

The results of the comparison made from EEG dataset using the above two methods is shown in Table 2. Rows show the different classes based on the 


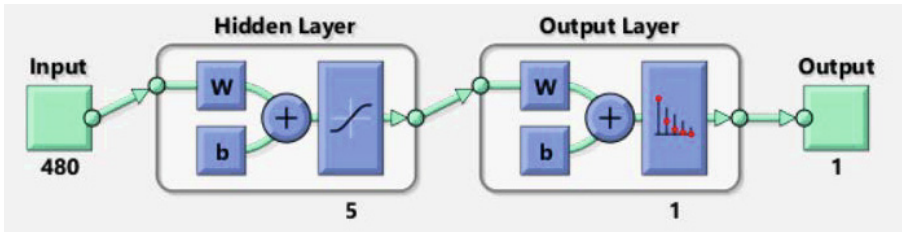

Fig. 4. Neural network configuration with 5 neurons in an unique hidden layer

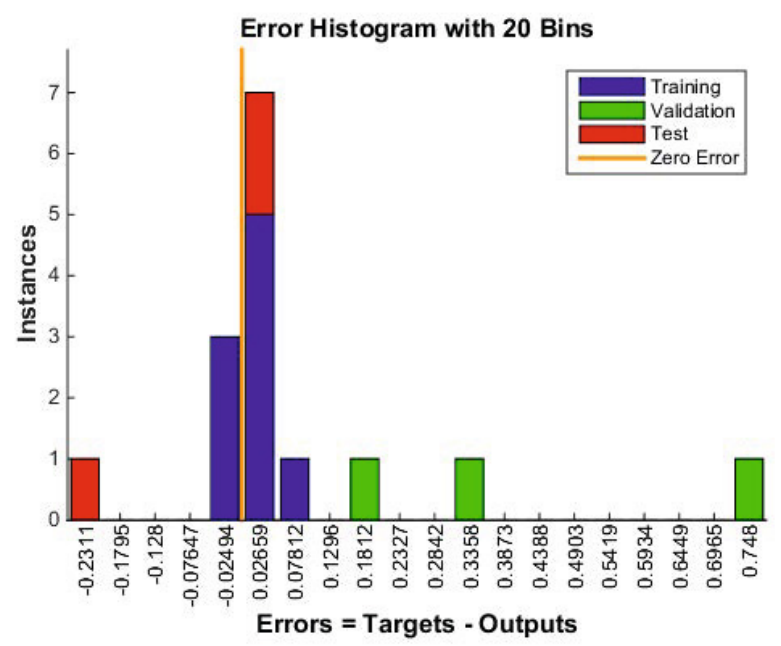

Fig. 5. Neural network training error histogram

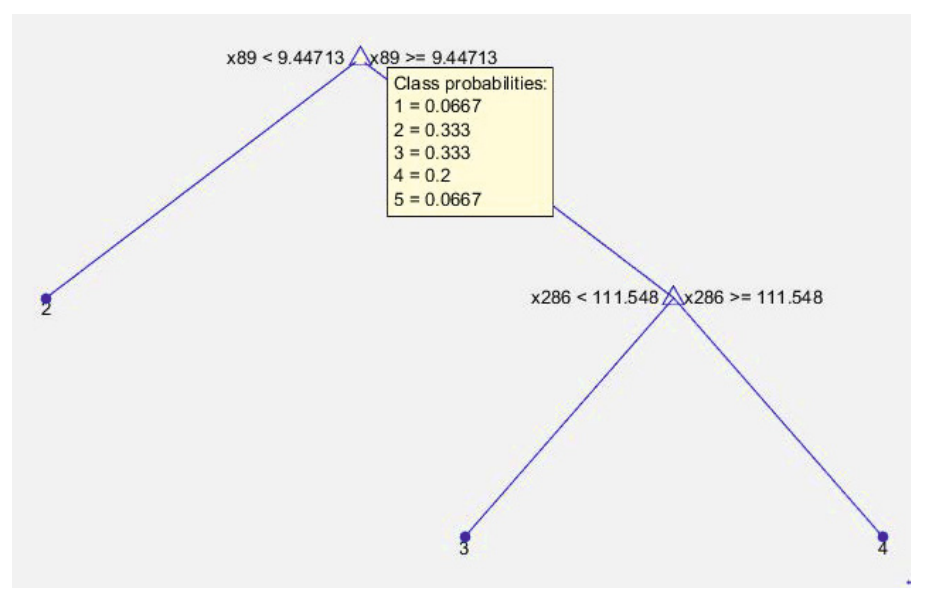

Fig. 6. Binary tree structure after the training process 
score assigned by users to each advertisement (1-5). Columns present the details associated with each classification methods employed (ANN and C4.5). First column shows the number of advertisement well-classified for each class using ANN. the second one presents the number of advertisements misclassified. Same items for results obtained from $\mathrm{C} 4.5$ algorithm are shown in the third and fourth column. Next column represents the number of advertisements of the dataset on each class (score). Finally, the accuracy obtained for ANN and C4.5 is presented in the last two columns.

Table 2. Classification results for short advertising movies from EEG inputs

\begin{tabular}{|c|c|c|c|c|c|c|c|}
\hline Class & $\begin{array}{l}\text { ANN suc- } \\
\text { cess }\end{array}$ & $\begin{array}{ll}\text { ANN er- } \\
\text { rors }\end{array}$ & $\begin{array}{l}\text { C4.5 suc- } \\
\text { cess }\end{array}$ & $\begin{array}{l}\text { C4.5 } \\
\text { errors }\end{array}$ & $\begin{array}{l}\text { Total in- } \\
\text { stances }\end{array}$ & $\begin{array}{l}\text { ANN } \\
\text { accuracy } \\
(\%)\end{array}$ & $\begin{array}{l}\mathrm{C} 4.5 \\
\text { accuracy } \\
(\%)\end{array}$ \\
\hline \begin{tabular}{|l|} 
Class 1 \\
\end{tabular} & 15 & 3 & 13 & 5 & 18 & 83 & 72 \\
\hline Class 2 & 18 & 4 & 16 & 6 & 22 & 81 & 72 \\
\hline Class 3 & 12 & 4 & 11 & 5 & 16 & 75 & 68 \\
\hline Class 4 & 27 & 6 & 22 & 11 & 33 & 81 & 66 \\
\hline Class 5 & 19 & 5 & 16 & 8 & 24 & 79 & 66 \\
\hline \begin{tabular}{|l|} 
Summary \\
\end{tabular} & 91 & 22 & 78 & 35 & 113 & 80 & 69 \\
\hline
\end{tabular}

\section{Future Works}

Future efforts can be oriented to improve the multimodal classification performance of this works. In one hand, data-driven approach, e.g., principal component analysis (Lin et al., 2009) and independent component analysis (Lin et al., 2010a), might be feasible to further elaborate the EEG spatio-spectral dynamics associated with implicit emotional responses. On the other hand, additional information could be included in the short advertising movies in order to determine low-level details influences on users liking.

\section{Conclusions}

In this paper a system of recognition of tastes on TV ads has made. The system proposed can automatically determine, through prior learning, whether users like the advertisements displayed. For this purpose a dataset consisting of over 400 statisticians from 11 basic signals obtained through electroencephalography device Neurosky Mindwave has been used. Subsequently a reduction process variables using PCA has conducted, resulting in a dataset of 15 variables to perform the statistical learning process. Two different algorithms, bye the one side artificial neural network and on the other side binary classification tree, have been used for the learning process. In the first case, an accuracy of approximately $80 \%$ was obtained, while in the second case the accuracy is lowered to $69 \%$ on average. Although the results are not excessively high, it is an important step towards inclusion of such systems on a daily life using low cost wearable sensors such as Neurosky Mindwave. 
Acknowledgments. This research is partially supported by the projects of the Spanish Ministry of Economy and Competitiveness HERMES (TIN2013-46801C4-1-r) and Simon(TIC-8052) of the Andalusian Regional Ministry of Economy, Innovation and Science.

\section{References}

[Lee et al.(2007)Lee, Broderick, and Chamberlain] Lee, N., Broderick, A.J., Chamberlain, L.: What is neuromarketing? A discussion and agenda for future research. International Journal of Psychophysiology 63, 199-204 (2007)

[Ariely and Berns(2010)] Ariely, D., Berns, G.S.: Neuromarketing: the hope and hype of neuroimaging in business. Nature Reviews Neuroscience 11, 284-292 (2010)

[Morin(2011)] Morin, C.: Neuromarketing: the new science of consumer behavior. Society 48, 131-135 (2011)

[Senior and Lee(2008)] Senior, C., Lee, N.: Editorial: A manifesto for neuromarketing science (2008)

[Murphy et al.(2008)Murphy, Illes, and Reiner] Murphy, E.R., Illes, J., Reiner, P.B.: Neuroethics of neuromarketing. Journal of Consumer Behaviour 7, 293-302 (2008)

[Hagan et al.(1996)Hagan, Demuth, Beale, et al.] Hagan, M.T., Demuth, H.B., Beale, M.H., et al.: Neural network design, vol. 1, Pws Boston (1996)

[Dreiseitl and Ohno-Machado(2002)] Dreiseitl, S., Ohno-Machado, L.: Logistic regression and artificial neural network classification models: a methodology review. Journal of Biomedical Informatics 35, 352-359 (2002)

[Quinlan(1993)] Quinlan, J.R.: C4. 5: programs for machine learning, vol. 1. Morgan kaufmann (1993)

[Vecchiato et al.(2011) Vecchiato, Astolfi, De Vico Fallani, Toppi, Aloise, Bez, Wei, Kong, Dai, Cincotti, et al.] Vecchiato, G., Astolfi, L., De Vico Fallani, F., Toppi, J., Aloise, F., Bez, F., Wei, D., Kong, W., Dai, J., Cincotti, F., et al.: On the use of EEG or MEG brain imaging tools in neuromarketing research. Computational Intelligence And Neuroscience 2011, 3 (2011)

[Alliance(2003)] Alliance, O.: Osgi service platform, release 3. IOS Press, Inc. (2003)

[Martín et al.(2009)Martín, Seepold, Madrid, Álvarez, Fernández-Montes, and Ortega] Martín, J., Seepold, R., Madrid, N.M., Álvarez, J.A., Fernández-Montes, A., Ortega, J.A.: A home e-Health System for Dependent people based on OSGi. In: Madrid, N.M., Seepold, R.E.D. (eds.) Intelligent Technical Systems. LNEE, vol. 38, pp. 117130. Springer, Heidelberg (2009)

[Martínez Fernández et al.(2010)Martínez Fernández, Seepold, Augusto, and Madrid] Fernández, J.M., Seepold, R., Augusto, J.C., Madrid, N.M.: Sensors in trading process: A Stress Aware Trader. In: IEEE 8th Workshop on Intelligent Solutions in Embedded Systems (WISES), pp. 17-22 (2010)

[Soria-Morillo et al.(2011)Soria-Morillo, Ortega-Ramírez, González-Abril, and ÁlvarezGarcía] Soria-Morillo, L.M., Ortega-Ramírez, J.A., González-Abril, L., ÁlvarezGarcía, J.A.: Mobile architecture for communication and development of applications based on context. In: Mehrotra, K.G., Mohan, C.K., Oh, J.C., Varshney, P.K., Ali, M. (eds.) IEA/AIE 2011, Part II. LNCS, vol. 6704, pp. 48-57. Springer, Heidelberg (2011) 